Draft Version November 30, 2017

Preprint typeset using $\mathrm{L}^{A} \mathrm{~T}_{\mathrm{E}} \mathrm{X}$ style emulateapj v. 01/23/15

\title{
Mg II ABSORBERS: METALLICITY EVOLUTION AND CLOUD MORPHOLOGY
}

\author{
Ting-Wen Lan ${ }^{1}$ and Masataka Fukugita ${ }^{1,2}$ \\ ${ }^{1}$ Kavli Institute for the Physics and Mathematics of the Universe, University of Tokyo, Kashiwa, Chiba 277-8583, Japan and \\ ${ }^{2}$ Institute for Advanced Study, Princeton, NJ 08540, U.S.A. \\ Draft version November 30, 2017
}

\begin{abstract}
Metal abundance and its evolution are studied for $\mathrm{Mg}$ II quasar absorption line systems from their weak, unsaturated spectral lines using stacked spectra from the archived data of Sloan Digital Sky Survey. They show an abundance pattern that resembles that of the Galactic halo or Small Magellanic Cloud, with metallicity $[\mathrm{Z} / \mathrm{H}]$ showing an evolution from redshift $z=2$ to 0.5 : metallicity becomes approximately solar or even larger at $z \approx 0$. We show that the evolution of the metal abundance traces the cumulative amount of the hydrogen fuel consumed in star formation in galaxies. With the aid of a spectroscopic simulation code, we infer the median gas density of the cloud to be roughly $0.3 \mathrm{~cm}^{-3}$, with which the elemental abundance in various ionization stages, in particular C I, is consistently explained. This gas density implies that the size of the Mg II clouds is of the order of $0.03 \mathrm{kpc}$, which suggests that individual $\mathrm{Mg}$ II clouds around a galaxy are of a baryonic mass typically $10^{3} \mathrm{M}_{\odot}$. This means that Mg II clouds are numerous and 'foamy', rather than a large entity that covers a sizable fraction of galaxies with a single cloud.
\end{abstract}

Keywords: quasars: absorption lines, galaxies: haloes

\section{INTRODUCTION}

Mg II quasar absorption clouds ubiquitously reside in the vicinity of galaxies, typically within their virial radii, in circumgalactic space (Bergeron \& Boissé 1991; Steidel et al. 1994). The absorption features at intervening redshifts are detected from 35-40\% of quasar spectra (e.g., Zhu \& Ménard 2013, for modern SDSS data) and MgII clouds cover as large as $50 \%$ of the sky around galaxies typically at redshift $\sim 0.5$ (e.g. Chen et al. 2010, Ménard \& Fukugita 2012, Nielsen et al. 2013; Lan et al. 2014). We expect the clouds affected by neighbouring galaxies, yet their nature and formation are not known well, for our knowledge of the cloud is limited to line of sight observations. The feature we now know is that MgII clouds are significantly contaminated with metals, habouring dust (e.g. York et al. 2006, Ménard \& Fukugita 2012), whereas star formation activity therein is not known, nor expected given the low column density of the clouds. The morphology of the clouds is also yet to be known: whether they consist of several large clouds that cover a significant fraction of galaxies, or an assembly of small clouds.

Elemental analyses for these clouds are often hampered by the fact that important metal lines are saturated, whereas weak lines suffer from poor signal to noise ratios to carry out a detailed analysis. In the present work we explore metal lines, using weak, unsaturated lines by stacking many spectra to give sufficient signal to noise ratios for those absorption lines. This enables us to derive elemental abundance as average quantities, as a function of the strength of spectral lines and redshift specified, and then the pattern of the elemental abundance, and/or its evolution.

One can also infer the physical state of the gas with the aid of a spectroscopic synthesis code, CLOUDY (Ferland et al. 2013), which reveals the physical state of the gas cloud for a given number density of atoms, metallicity and ionising fields. This, after verifying the validity of the code for the problems that concern us, would lead us to infer physics of the clouds. This in turn hints us to infer their morphology around galaxies.

Our data analysis is written in Section 2 to measure the equivalent width, with some results in our intermediate steps given in Appendix. We discuss in Section 3 the column density of elements that show absorption features in MgII clouds. The CLOUDY code was employed to infer the physical state of the gas in the clouds. In this work all equivalent widths referred to are those in the rest frame. Wherever we refer to the average value, we take median quantities to avoid largely deviated data unless otherwise explicitly stated. When we refer to the solar composition, it is that by Asplund et al. (2009). Section 4 is given for the summary of our analysis. We use $H_{0}=70$ $\mathrm{km} \mathrm{s}^{-1} \mathrm{Mpc}^{-1}$, and $\Omega_{\mathrm{M}}=0.3$ in a flat Universe. $W_{\lambda 2796}$ refers to the rest equivalent width of MgII $\lambda 2796$ line.

\section{DATA ANALYSIS}

\subsection{Composite spectra for metal absorption lines}

We use the metal absorber catalogud 1 and the corresponding spectra compiled by Zhu \& Ménard (2013) from quasar spectra of the Sloan Digital Sky Survey 1-III (York et al. 2000). The sample contains 77,647 MgII absorbers from redshift 0.4 to 2.5 , detected in 142,012 quasar spectra from the DR7 (Schneider et al. 2010) and DR12 (Pâris et al. 2017) quasar catalogues. In the present study, we take 70,713 systems with $W_{\lambda 2796}>0.4 \AA$. The completeness of MgII absorbers drops gradually from $W_{\lambda 2796}=0.8 \AA$ and it is about $30 \%$ at $W_{\lambda 2796}=0.4 \AA$. The completeness is not an important issue in major parts of our analysis.

We make median composite spectra of MgII absorbers divided into bins of their absorption strengths, as char-

1 jhu-sdss-metal-absorber-catalog http://www.guangtunbenzhu.com/ 


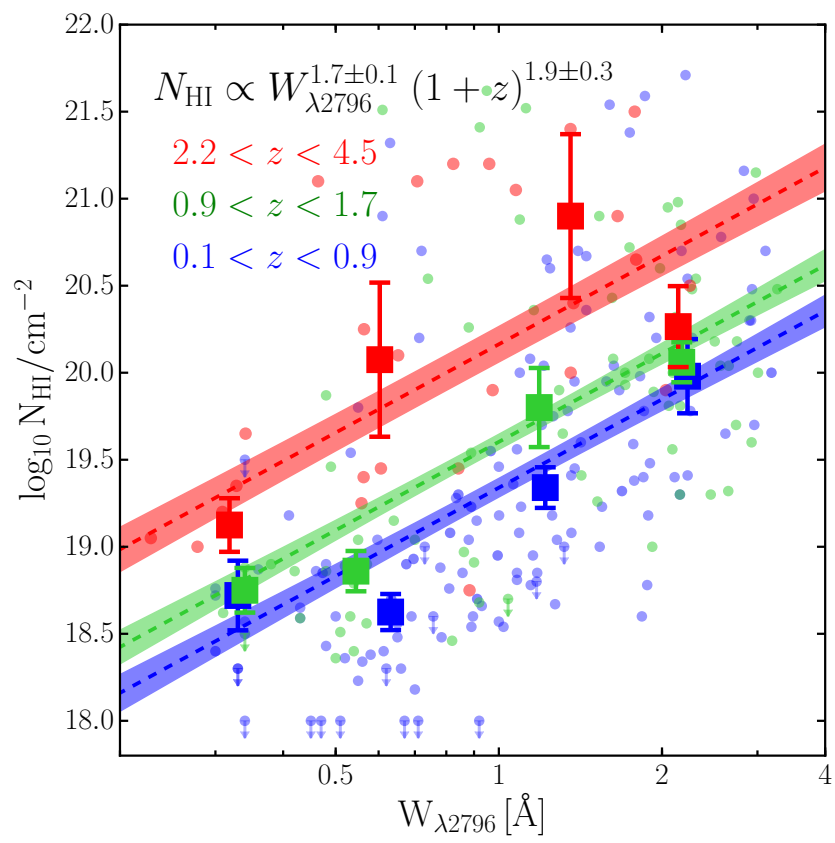

Figure 1. $N_{\mathrm{HI}}$ versus MgII equivalent widths of the clouds. Symbols with three colours represent samples in different redshifts, as shown in the legend with the redshift ranges presented. Square data points are median values of the three samples with bootstrapping errors. Three lines are fit to the data with equation (2) using common $\alpha$ and $\beta$, as explained in the text.

acterized with $\operatorname{MgII} \lambda 2796$, and redshifts. We focus on rest-frame wavelengths longer than $1250 \AA$. The median estimator is used to avoid too strong effects of outliers in spectra that occasionally occur. We emphasize that it is essential to our work to measure weak unsaturated absorption lines accurately.

The metal absorption lines we measure are listed in Table 1 below. The typical signal-to-noise ratio of the composite spectra per spectral resolution element $(70 \mathrm{~km} / \mathrm{s})$ is about 500 that allows us to measure absorption features to the level of $0.01 \AA$ in equivalent widths.

We measure the rest-frame equivalent widths of isolated metal lines with a single Gaussian profile fitting. For wavelength regions with multiple lines crowded, we adopt multiple Gaussian profiles to fit all relevant absorption lines in both vicinity of lines and continuum. A special treatment is used to obtain the rest equivalent widths of $\mathrm{ZnII}$ to deblend lines of $\mathrm{CrII}$ and $\mathrm{MgI}$ (e.g., York et al. 2006). The errors of the rest equivalent widths are estimated by bootstrapping the sample for 200 times. In Appendix, we show an example of the composite spectra, the Gaussian profile fitting and the measured rest equivalent widths of metal lines as a function of redshift and MgII strengths.

For weak absorption lines we can estimate the column density with the linear relation of the curve of growth (e.g., Draine 2011),

$$
N_{\text {ion }}\left[\mathrm{cm}^{-2}\right]=1.13 \times 10^{20} \times \frac{W_{\text {ion }}}{f \lambda^{2}},
$$

where the oscillator strength $f$ is from Morton (2003) and $\lambda(\AA)$ the rest-frame wavelength of the absorption line. For weak unsaturated lines we give in Table 1 (Column 3 ) the estimated column density. By comparing measure- ments with the theoretical curve of growth, we confirm that those absorption lines are in the linear regime. The strong, saturated absorption lines are marked in Column (3) with an asterisk.

\section{RESULTS}

\subsection{Evolution of the HI column density in MgII absorbers}

Let us first study neutral hydrogen in MgII absorbers. We take additionally Mg II absorber samples with neutral hydrogen column densities measured with the Voigt fitting to study their HI content: (i) The $z \sim 1$ sample from Rao et al. (2006), which consists of $197 \mathrm{MgII}$ absorbers at $z<1.7$ with $N_{\mathrm{HI}} \geq 10^{18} \mathrm{~cm}^{-2}$; (ii) high redshift $(z \sim 3)$ sample from Matejek et al. (2013), containing 33 systems with median redshift about 3.4. These are supplemented by (iii) the recent low redshift $(z \sim 0.3)$ sample of $16 \mathrm{Mg}$ II absorbers in Rao et al. (2017).

Figure 1 shows the samples in the order of blue symbols $(\langle z\rangle \simeq 0.6)$, green $(\langle z\rangle \simeq 1.2)$, and red $(\langle z\rangle \simeq 3.4)$, taken from the references cited above. We also show the median values of the samples with bootstrapping errors shown with square data points. We observe that the column densities of neutral hydrogen increase with the equivalent widths of $\mathrm{MgII}$ absorption lines ( $\mathrm{MgII} \lambda 2796)$, and at a fixed MgII absorption strength monotonically with redshifts, as noted in Ménard \& Chelouche (2009) and Matejek et al. (2013).

These trends are summarised as

$$
N_{\mathrm{HI}}=A\left(\frac{W_{\lambda 2796}}{1 \AA}\right)^{\alpha}(1+z)^{\beta},
$$

where $W_{\lambda 2796}$ refer to $\operatorname{MgII} \lambda 2796$. From the fits to three separate samples for different redshift bins we find that slopes for the $W_{\lambda 2796}$ dependence are mutually consistent within errors. We, therefore, adopt a common parameter $\alpha$ for the slope for our full sample analysis. To make use of all the information, we obtain the final best-fit parameter values through a fitting to all the individual data points $\left(\log N_{\mathrm{HI}}, W_{\lambda 2796}, z\right)^{2}$

The neutral hydrogen column densities increase with the MgII equivalent width with a power index $\alpha=1.69 \pm$ 0.13 . For the other parameters we find $\beta=1.88 \pm 0.29$, and $A=10^{18.96 \pm 0.10} \mathrm{~cm}^{-2}$, which are also given in Table 1. The errors are estimated by bootstrapping the samples for 200 times. The fits are presented in Figure 1 for the three redshift bins, with median redshifts $0.6,1.2$, and 3.4 .

\subsection{Metals in MgII absorbers}

Having measured the rest-frame equivalent width of each absorption line for the composite spectrum for bins of subsample where the MgII equivalent width and the redshift specified, we fit to our data with

$$
W_{\lambda}=C\left(\frac{W_{\lambda 2796}}{1 \AA}\right)^{\alpha}(1+z)^{\beta},
$$

2 We have obtained the best-fit parameter values with three methods,(1) fitting to all the individual data points, (2) fitting to median values with bins, and (3) fitting to mean values with bins, and confirmed that the three methods yield consistent bestfit parameters. 
Table 1

Best fit parameters for Equation 3, $W_{\lambda}=C\left(W_{\lambda 2796}\right)^{\alpha}(1+z)^{\beta}$

\begin{tabular}{|c|c|c|c|c|c|}
\hline Transition & wavelength $[\AA]$ & $N$ column density $^{a}\left[10^{12} \mathrm{~cm}^{-2}\right]$ & $\alpha$ & $\beta$ & $C[\AA]$ \\
\hline HI & 1215.67 & $10^{6.96 \pm 0.10}$ & $1.69 \pm 0.13$ & $1.88 \pm 0.29$ & \\
\hline SiII & 1260.42 & ${ }^{*}$ Saturated ${ }^{b}$ & $1.07 \pm 0.01$ & $-0.22 \pm 0.13$ & $0.573 \pm 0.086$ \\
\hline OI & 1302.17 & $*$ & $1.48 \pm 0.02$ & $0.28 \pm 0.17$ & $0.145 \pm 0.028$ \\
\hline SiII & 1304.37 & * & $1.35 \pm 0.03$ & $-0.62 \pm 0.19$ & $0.325 \pm 0.069$ \\
\hline NiII & 1317.22 & $0.4 \pm 1.0$ & $1.53 \pm 0.29$ & $3.25 \pm 2.16$ & $0.000 \pm 0.001$ \\
\hline CII & 1334.53 & * & $1.05 \pm 0.01$ & $-0.27 \pm 0.07$ & $0.635 \pm 0.051$ \\
\hline NiII & 1370.13 & $42.8 \pm 55.5$ & $1.45 \pm 0.18$ & $-1.41 \pm 1.14$ & $0.055 \pm 0.071$ \\
\hline SiIV & 1393.76 & $*$ & $0.66 \pm 0.01$ & $-1.01 \pm 0.10$ & $1.124 \pm 0.122$ \\
\hline SiIV & 1402.77 & $*$ & $0.65 \pm 0.02$ & $-1.05 \pm 0.13$ & $0.793 \pm 0.111$ \\
\hline NiII & 1454.84 & $23.3 \pm 49.4$ & $1.60 \pm 0.36$ & $-0.99 \pm 2.00$ & $0.014 \pm 0.030$ \\
\hline SiII & 1526.71 & $*$ & $1.35 \pm 0.01$ & $-0.25 \pm 0.05$ & $0.313 \pm 0.016$ \\
\hline CIV & 1548.20 & * & $0.58 \pm 0.01$ & $-0.87 \pm 0.05$ & $1.524 \pm 0.081$ \\
\hline CIV & 1550.78 & * & $0.60 \pm 0.01$ & $-1.30 \pm 0.06$ & $1.728 \pm 0.104$ \\
\hline CI & 1560.31 & $56.6 \pm 65.6$ & $1.99 \pm 0.36$ & $-3.09 \pm 1.10$ & $0.094 \pm 0.109$ \\
\hline FeII & 1608.45 & $*$ & $1.70 \pm 0.02$ & $-0.76 \pm 0.09$ & $0.165 \pm 0.016$ \\
\hline CI & 1656.93 & $124.3 \pm 55.2$ & $1.97 \pm 0.13$ & $-3.86 \pm 0.45$ & $0.449 \pm 0.200$ \\
\hline AlII & 1670.79 & $*$ & $1.33 \pm 0.01$ & $-0.33 \pm 0.03$ & $0.375 \pm 0.012$ \\
\hline NiII & 1709.60 & $9.5 \pm 4.4$ & $1.48 \pm 0.11$ & $0.11 \pm 0.45$ & $0.008 \pm 0.004$ \\
\hline NiII & 1741.55 & $23.6 \pm 6.7$ & $1.52 \pm 0.07$ & $-0.70 \pm 0.28$ & $0.027 \pm 0.008$ \\
\hline NiII & 1751.92 & $29.8 \pm 11.1$ & $1.45 \pm 0.10$ & $-0.78 \pm 0.36$ & $0.022 \pm 0.008$ \\
\hline SiII & 1808.01 & $562.3 \pm 89.1$ & $1.67 \pm 0.04$ & $-0.50 \pm 0.15$ & $0.034 \pm 0.005$ \\
\hline AlIII & 1854.72 & $*$ & $1.19 \pm 0.01$ & $-0.85 \pm 0.05$ & $0.255 \pm 0.012$ \\
\hline AlIII & 1862.79 & $16.8 \pm 1.3$ & $1.24 \pm 0.02$ & $-0.86 \pm 0.08$ & $0.143 \pm 0.011$ \\
\hline ZnII & 2026.14 & $1.4 \pm 0.3$ & $1.96 \pm 0.08$ & $-1.10 \pm 0.20$ & $0.025 \pm 0.005$ \\
\hline CrII & 2056.26 & $5.8 \pm 1.2$ & $1.47 \pm 0.08$ & $-0.69 \pm 0.23$ & $0.022 \pm 0.005$ \\
\hline ZnII & 2062.66 & $1.4 \pm 0.5$ & $2.15 \pm 0.18$ & $-1.22 \pm 0.41$ & $0.013 \pm 0.005$ \\
\hline CrII & 2066.16 & $5.4 \pm 2.5$ & $1.36 \pm 0.16$ & $-0.71 \pm 0.48$ & $0.010 \pm 0.005$ \\
\hline FeII & 2249.88 & $325.3 \pm 44.5$ & $1.55 \pm 0.06$ & $-0.81 \pm 0.15$ & $0.027 \pm 0.004$ \\
\hline FeII & 2260.78 & $313.6 \pm 33.5$ & $1.59 \pm 0.04$ & $-0.83 \pm 0.12$ & $0.035 \pm 0.004$ \\
\hline FeII & 2344.21 & $*$ & $1.61 \pm 0.01$ & $-0.56 \pm 0.02$ & $0.353 \pm 0.006$ \\
\hline FeII & 2374.46 & $*$ & $1.71 \pm 0.01$ & $-0.66 \pm 0.03$ & $0.179 \pm 0.005$ \\
\hline FeII & 2382.77 & $*$ & $1.44 \pm 0.00$ & $-0.43 \pm 0.01$ & $0.533 \pm 0.006$ \\
\hline MnII & 2576.88 & $2.4 \pm 0.2$ & $1.69 \pm 0.04$ & $-1.19 \pm 0.09$ & $0.052 \pm 0.004$ \\
\hline FeII & 2586.65 & $*$ & $1.65 \pm 0.01$ & $-0.60 \pm 0.02$ & $0.317 \pm 0.005$ \\
\hline MnII & 2594.50 & $1.7 \pm 0.2$ & $1.52 \pm 0.06$ & $-0.78 \pm 0.14$ & $0.028 \pm 0.004$ \\
\hline FeII & 2600.17 & $*$ & $1.48 \pm 0.00$ & $-0.45 \pm 0.01$ & $0.534 \pm 0.006$ \\
\hline MnII & 2606.46 & $2.0 \pm 0.3$ & $1.74 \pm 0.08$ & $-1.14 \pm 0.17$ & $0.024 \pm 0.004$ \\
\hline MgII & 2796.35 & $*$ & $1.00 \pm 0.00$ & $0.00 \pm 0.00$ & $1.000 \pm 0.001$ \\
\hline MgII & 2803.53 & $*$ & $1.11 \pm 0.00$ & $-0.01 \pm 0.01$ & $0.797 \pm 0.004$ \\
\hline MgI & 2852.96 & $1.6 \pm 0.0$ & $1.45 \pm 0.01$ & $-0.67 \pm 0.03$ & $0.215 \pm 0.005$ \\
\hline TiII & 3242.92 & $0.3 \pm 0.1$ & $1.55 \pm 0.15$ & $-0.15 \pm 0.38$ & $0.007 \pm 0.002$ \\
\hline TiII & 3384.73 & $0.4 \pm 0.1$ & $1.51 \pm 0.09$ & $-0.31 \pm 0.26$ & $0.015 \pm 0.003$ \\
\hline CaII & 3934.77 & $1.2 \pm 0.1$ & $1.53 \pm 0.03$ & $-0.77 \pm 0.12$ & $0.099 \pm 0.008$ \\
\hline CaII & 3969.59 & $1.8 \pm 0.2$ & $1.57 \pm 0.06$ & $-1.32 \pm 0.20$ & $0.076 \pm 0.010$ \\
\hline $\mathrm{E}_{\mathrm{g}-\mathrm{i}}$ & & $0.017 \pm 0.003[\mathrm{mag}]$ & $1.6 \pm 0.1$ & $-1.2 \pm 0.2$ & \\
\hline$E_{B-V}$ & & $0.011 \pm 0.002[\mathrm{mag}]$ & $1.6 \pm 0.1$ & $-1.2 \pm 0.2$ & \\
\hline
\end{tabular}

${ }^{a} \mathrm{~N}$ column density values are estimated from the $\mathrm{C}$ parameter values in column 6 with Eq. 1.

${ }^{b}$ Saturated lines are marked with *

where $\alpha, \beta$ and $C$, are given in Table 1 , and also displayed in Figure 2, where the plots are basically divided into two classes, saturated (red) and unsaturated lines (blue). The green symbols stand for the parameters for HI obtained above and that for dust reddening parametrised in a similar way as to $\alpha$ and $\beta$, i.e., $E(B-V)$ in place of $W_{\lambda}$ written in the form as equation (3) (Ménard et al. 2008: Ménard \& Fukugita 2012 ${ }^{3}$, We show the measured rest equivalent widths and the best-fit functions in Appendix.

3 The fit here refers to the rest-frame colour, as in Ménard et al. (2008), while a similar fit of Ménard \& Fukugita (2012) uses the colour in the observed frame. The power indices of the two differ by 1.2 , reflecting the $\lambda^{-1.2}$ dependence of the extinction curve.
The following trends are seen in Figure 2:

- All metal absorption lines (with an exception of OI) show negative $\beta$, meaning an absorption stronger at lower redshift when measured at a fixed MgII equivalent width. This indicates the equivalent widths, or column densities in case of unsaturated lines, decrease approximately, in median, by a factor of 1.6 from redshift 0.5 to 2 .

This contrasts to neutral hydrogen, a larger equivalent width at higher redshift $(\beta \simeq 1.9)$.

- Most of the low-ionized, unsaturated absorption lines show $\alpha=1.4-1.7$ with a median $\sim 1.6$, which 


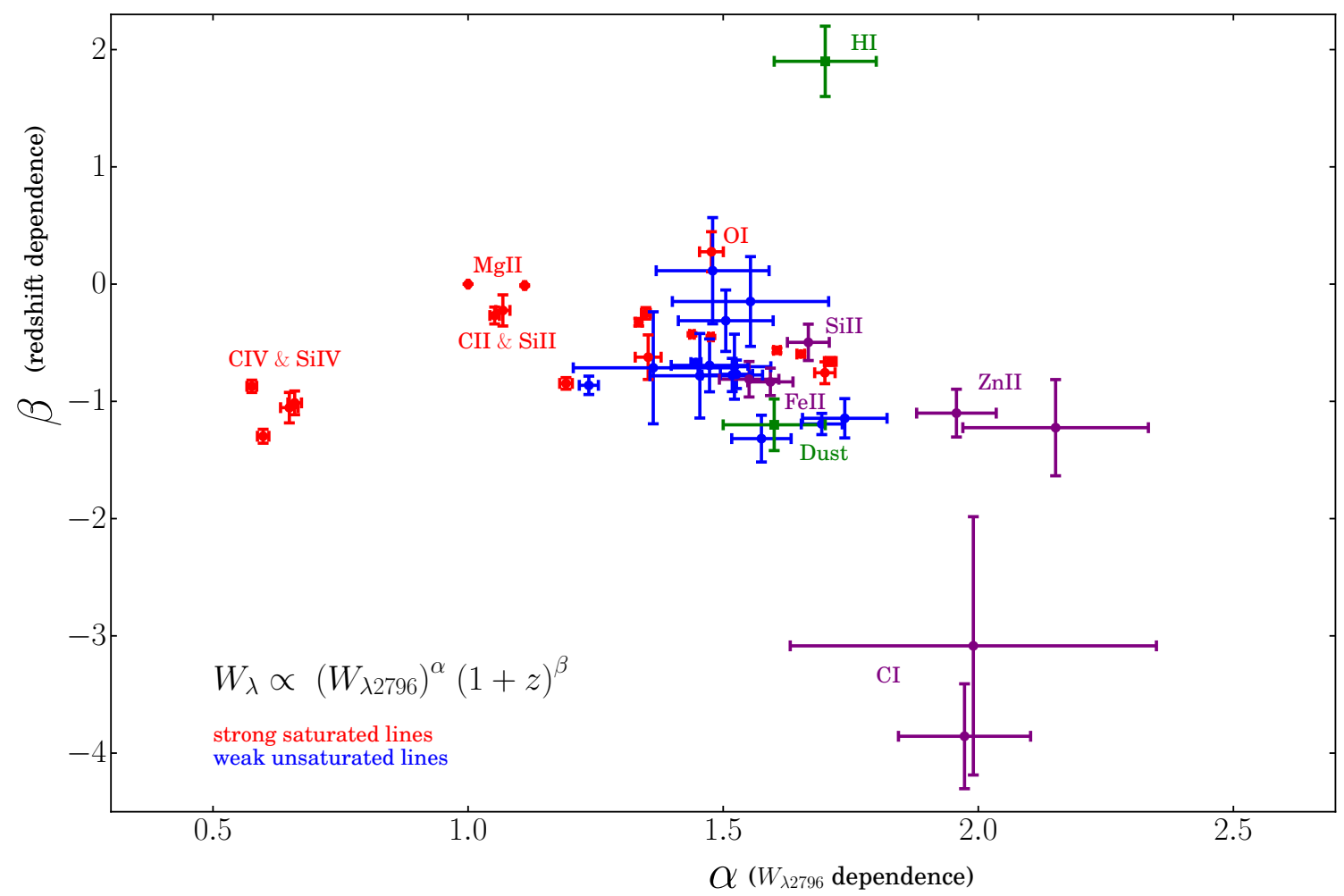

Figure 2. Dependence of the equivalent width of each absorption line upon the strength of MgII absorption line (MgII $\lambda 2796), \alpha$, and redshift, $\beta$, as parametrized in equation (3). Blue symbols indicate weak unsaturated lines, red symbols are for saturated lines. Purple symbols highlight Si II, FeII, ZnII and C I which are discussed in the text. Green symbols are used for the hydrogen column density and for extinction $E(B-V)$.

is consistent with $\alpha$ for $N_{\mathrm{HI}}$, Namely the abundances of metals in line of sight is proportional to neutral hydrogen column density, or in other words, metallicity of MgII clouds does not vary much with $W_{\lambda 2796}$.

We remark that $\alpha$ of $\mathrm{ZnII}$ is somewhat larger, $\alpha \sim$ 2 , which is about $3 \sigma$ away from the remainders.

- CI absorption shows both $\alpha$ and $\beta$ largely different from other lines. The $\beta \sim-3.9$ value indicates that the CI equivalent width decreases by more than a factor 5 from redshift 1 to 2.5. We will pay a special attention to CI below.

- Low-ionized saturated absorption lines, MgII, CII, SiII are nonevolving: $\beta \approx 0$. The $\alpha$ values being close to 1 means a similarity to our reference MgII absorption line. This is expected for the saturated rest equivalent width which is controlled by the velocity dispersion of the system.

- Highly-ionized absorption lines, CIV and SiIV, are saturated. They show $\alpha \sim 0.6$ and $\beta \sim-1$ that differ from low-ionised saturated lines.

- We added $E(B-V)$ for a reference, taken from Ménard \& Fukugita (2012). $\alpha \simeq 1.6$ means $E(B-$ $V) \propto N_{\mathrm{HI}}$, and $\beta \sim-1.2$ is consistent with the decrease of metallicity to higher $z$.

In Figure 3 we show the evolution of the abundance pattern for several representative heavy elements in single ionized state in MgII absorbers with $W_{\lambda 2796}>0.8 \AA$, as a function of redshift, taking the column density of $\mathrm{ZnII}$ as the reference. At this stage we do not apply ionisation corrections, which turn out to be appreciable for Zn II, to facilitate their comparison with elemental abundance in the literature, in which ionisation corrections are usually not made. The zero point of the ordinate is the solar. Horizontal dashed lines show the abundance taken from York et al. (2006) representing the value in Milky Way's halo, which is similar to the SMC abundance (Welty et al. 2001), and that of cold interstellar matter. The figure shows that the abundance in MgII clouds is similar to that in Milky Way halo, in agreement with what was argued in York et al. The abundance is not in agreement with that of the interstellar medium of the Milky Way. It deviates from the solar, but they become close if they are shifted by 0.5 dex (0.2 dex for Si II) upwards. The relative abundance is also listed in Table 2. The upper part shows values without ionization correction and the lower part lists values for MgII clouds with ionization correction taking into account, which will be discussed in Section 3.3 below.

The 0.5 dex smaller abundance of the iron group elements, is likely to be ascribed to the condensation into grains. Si is also smaller than solar by $0.2 \mathrm{dex}$, as is the abundance in the halo, which is also ascribed to depletion onto grains. The metal abundance relative to $\mathrm{Zn}$ evolves weakly in the redshift range we study.

The lower rightmost panel shows the dust abundance divided by the $\mathrm{Zn}$ II abundance, where $E_{B-V} \simeq$ $0.01\left(W_{2796} / 1 \AA\right)^{1.6}(1+z)^{-1.2}$ is used for the proxy for dust (Ménard et al. 2008, Ménard \& Fukugita 2012). 


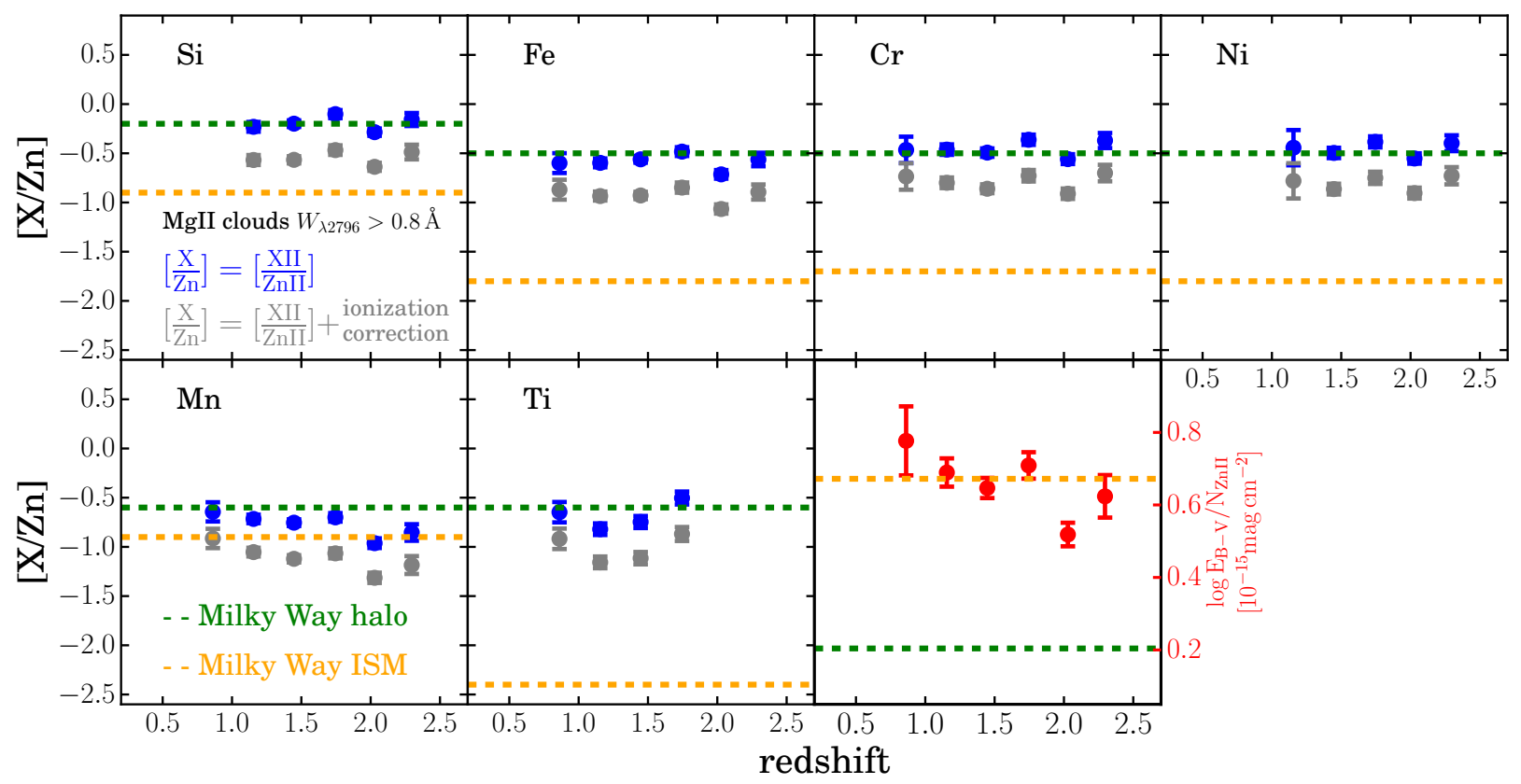

Figure 3. Abundance of each element relative to $\mathrm{Zn}$ as a function of redshift. The last panel shows the dust abundance as estimated from $E(B-V)$ divided by the column density of ZnII. Horizontal lines indicate the corresponding value for Milky Way halo (green) and for interstellar matter (orange) (York et al. 2006). The zero point is the solar value. Blue points refer to the relative abundance with no ionization correction, $[\mathrm{X} / \mathrm{Zn}]=[\mathrm{X} I \mathrm{I} / \mathrm{ZnII}]$ and grey points refer to the relative abundance after the ionisation corrections taken into account.

Table 2

Relative abundance patterns of MgII clouds, Milky Way halo, SMC, and Milky Way ISM

\begin{tabular}{|c|c|c|c|c|c|c|c|c|c|}
\hline$[\mathrm{X} / \mathrm{Zn}]=[\mathrm{XII} / \mathrm{ZnII}]$ & $\mathrm{C}$ & $\overline{\mathrm{Si}}$ & $\mathrm{Mg}$ & $\mathrm{Fe}$ & $\mathrm{Cr}$ & $\mathrm{Ni}$ & $\overline{\mathrm{Mn}}$ & $\mathrm{Ti}$ & Reference \\
\hline MgII clouds & 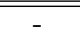 & 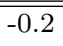 & "- & $\overline{-0.5}$ & $\overline{-0.5}$ & $\overline{-0.5}$ & $\begin{array}{l}-0.7 \\
\end{array}$ & 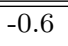 & This paper \\
\hline Milky Way halo & -0.2 & -0.2 & -0.2 & -0.5 & -0.5 & -0.5 & -0.6 & -0.6 & York et al. (2006) \\
\hline SMC (sk 108) & - & 0.1 & - & -0.5 & -0.5 & -0.8 & -0.6 & - & Welty et al. (2001) \\
\hline Milky Way cool ISM & 0.1 & -0.9 & -0.8 & -1.8 & -1.7 & -1.8 & -0.9 & -2.4 & York et al. (2006) \\
\hline $\begin{array}{c}\text { X/Zn }]=[\mathrm{XII} / \mathrm{ZnII}]+\text { ionization } \text { correction }^{\mathrm{a}} \\
\text { MgII clouds }\end{array}$ & $-0.6^{b}$ & -0.6 & $-0.6^{b}$ & -0.9 & -0.9 & -0.9 & -1.1 & -1.0 & This paper \\
\hline
\end{tabular}

${ }^{a}$ The ionization correction for Zinc, $\log N_{\mathrm{Zn}} \approx \log N_{\mathrm{ZnII}}+0.4$, is estimated with $\log \mathrm{n}_{\mathrm{H}}=-0.5$.

${ }^{b}$ Assuming Milky Way halo values with ionization correction

This dust to metal in gas ratio is close to the Milky Way's value (Wild et al. 2006), but is significantly larger than the values in SMC or in Milky Way halo by a factor of 3 .

\subsection{Physical conditions and ionisation corrections}

We have derived the column density of metals in their ionised state. For Fe II, Si II etc., these are predominant ionisation states and they represent practically the full column density of those heavy elements. For other states, however, we must know the physical state to infer how much fractions are in specific ionisation stages. When compared with the observation, this in turn tells us about the physical conditions of MgII absorbers. The other uncertainty arises from condensation into grains, as seen for iron and silicon.

We employ the CLOUDY code $\AA^{4}$ Ferland et al. 2013) to infer the ionisation correction factor for the given physical condition. We take the hydrogen column density

\footnotetext{
4 http://trac.nublado.org/wiki
}

$N_{\mathrm{H}}$, the volume density of hydrogen $n_{\mathrm{H}}$, and metallicity of gas as parameters. We set the relative element abundance pattern to the Milky Way halo value listed in Table 2. We find that the ionisation correction for ZnII is appreciable, while it is not for FeII, MgII etc.

We assume that the cloud is photoionised by the background radiation field, using the 2005 version of Haardt \& Madau cosmic background radiation field at each redshift, as in the default setting in CLOUDY 5 . We constrain CLOUDY simulations with the neutral hydrogen column density consistent with MgII absorbers as observed, and explore $n_{\mathrm{H}}$ and metallicity that repro-

5 This is an unpublished update of their 2001 version Haardt \& Madau 2001). There is an alternative choice of the cosmic background radiation field Haardt \& Madau (2012), which gives a photon spectrum somewhat tilted from their 2005 version. We have also carried out our analysis with the radiation field of Haardt \& Madau (2012), which yielded metallicity by $0.2-0.3$ dex higher $\left(n_{\mathrm{H}}\right.$ is lower by $0.3 \mathrm{dex}$ ). Because of this resulting supersolar metallicity, that looks unphysical, we do not take this 2012 version as our setting. 


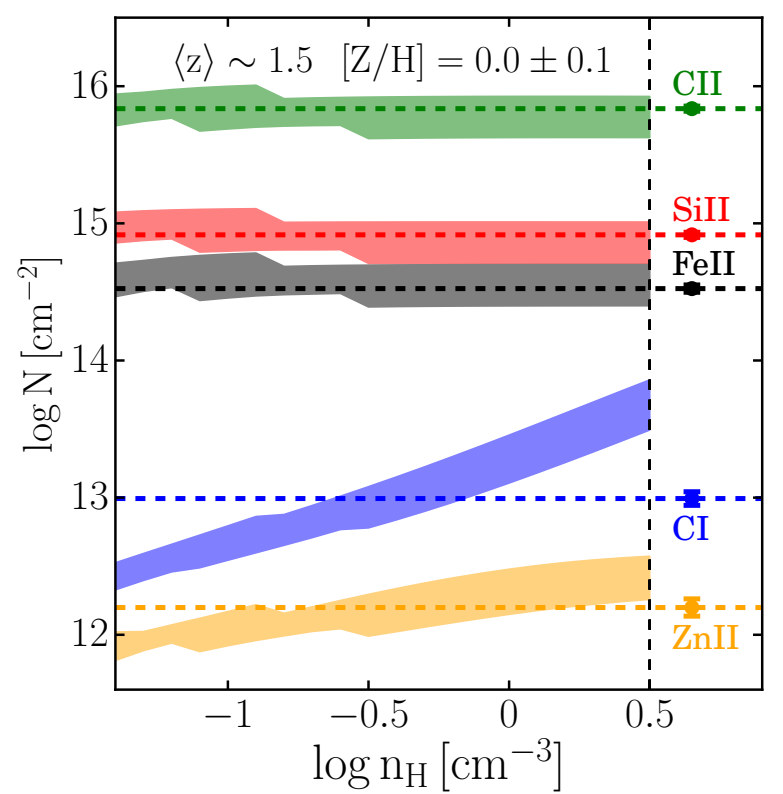

Figure 4. Column density of various species: comparisons are made of the observed values (the symbols in the rightmost and horizontal lines extended therefrom) against the CLOUDY simulation plotted with varying hydrogen volume densities.

duce the column densities of FeII, NiII, CI, Al III, CrII, $\mathrm{ZnII}$, and MgI as we observed. To constrain the physical conditions, we use measurements with high $\mathrm{S} / \mathrm{N}$ derived from composite spectra with MgII absorbers with $W_{\lambda 2796}>0.8 \AA$ in this section. We have also carried out the analysis as a function of $W_{\lambda 2796}$ and confirmed that the inferred physical conditions have a weak dependence with $W_{\lambda 2796}$.

Figure 4 shows an example of several metal column densities (SiII, FeII, CI, CII, ZnII) at $z \simeq 1.5$ from CLOUDY as a function of the gas density $n_{\mathrm{H}}$, compared with the observed values. Solar metallicity $[\mathrm{Z} / \mathrm{H}]=0$ is assumed. The observed column densities are also indicated by horizontal lines, together with the observation shown in the right margin. We see that the calculation becomes close to the observation for almost all elements we consider when $-1<\log n_{\mathrm{H}}<0$. We carry out this analysis at redshifts between $z=1$ and 2.5. We find that features of the curves of the metal column density versus $n_{\mathrm{H}}$ change only at a quantitative level in this redshift interval. We note that CI is particularly sensitive to the hydrogen volume density of the system, and also to redshift. Let us note that CI is a minor component while CII is the predominant agent of carbon.

We attempt to constrain simultaneously the volume density and the metallicity with the observed ZnII column density and the ratio of $N_{\mathrm{CI}}$ to $N_{\mathrm{CII}}$, which is estimated from $N_{\text {SiII }}$, as shown in Figure 5 for one and two sigma contours at $z \simeq 1.5$. The hydrogen volume density is about $\log n_{\mathrm{H}} \sim-0.5$ and metallicity consistent with the solar.

In Figure 6 we show the redshift evolution of CI from CLOUDY, which shows a rapid decrease with redshift, where we assume $\log n_{\mathrm{H}}=-0.5$ for all redshifts. The rapid evolution of CI agrees very well with that we derived from the observation.

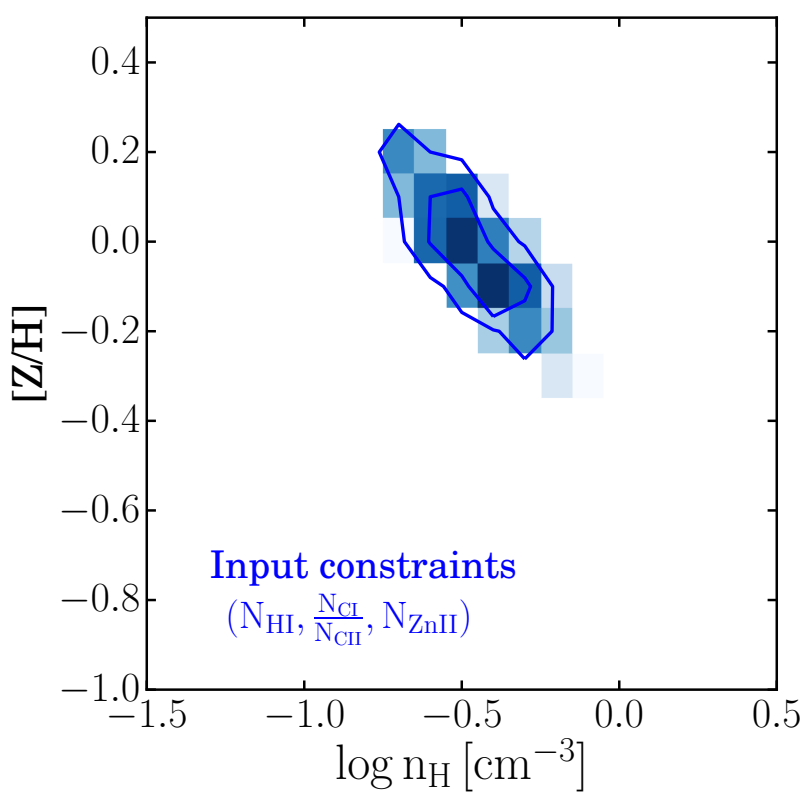

Figure 5. Allowed region for metallicity $[\mathrm{Z} / \mathrm{H}]$ and the gas density $\log n_{\mathrm{H}}$ at redshift $z \simeq 1.5$ constrained by the observed $N_{\mathrm{HI}}, \frac{N_{\mathrm{CI}}}{N_{\mathrm{CII}}}$, and $N_{\mathrm{ZnII}}$.

We then estimate the major component, CII abundance $N_{\text {CII }}$ from $N_{\text {CI }}$ using the ionisation correction factor. The CII column density thus calculated is nearly constant in our redshift range, where the ionisation correction factor for CI varies rapidly between 100 and 1000 . We compare it with $N_{\text {CII }}$ estimated from $N_{\text {SiII }}$ with the Milky Way halo abundance ratio of carbon to silicon. The agreement of the two estimates is impressive, verifying the validity of CLOUDY results with the 2005 version of the Haardt \& Madau ionisation field at each redshift.

We now lift the assumption of $\log n_{\mathrm{H}}=-0.5$ for all redshifts, and obtain the best fit at each redshift bin using $N_{\mathrm{HI}}, N_{\mathrm{CI}} / N_{\mathrm{CII}}$ and $N_{\mathrm{ZnII}}$ as constraints. Figure 7 shows $\log n_{\mathrm{H}}=-0.5_{-0.1}^{+0.2}$ at all redshifts that concern us. This indicates that a strong redshift evolution of CI column density, $N_{\mathrm{CI}} \propto(1+z)^{-3.9}$, is induced most importantly by a decrease of photoionising radiation field towards lower redshifts. This fast evolution of CI column density accounts for a rapid evolution of the CI cloud incidence, a rapid increase from $\mathrm{z}=2.5$ to 1.5 observed in Ledoux et al. (2015).

We also study the prediction of $\mathrm{Al}$ III, ZnII and $\mathrm{Mg}$ I column densities. We find that the observed Al III column density is consistent with the prediction for $-1 \leq$ $\log n_{\mathrm{H}} \leq-0.5$. The CLOUDY result for Zn II is consistent with the observation in so far as $-1 \leq \log n_{\mathrm{H}} \leq 0.5$. So, our choice, $\log n_{\mathrm{H}} \approx-0.5$, from $\mathrm{C}$ I is a compromise, consistent with the observation for Al III and Zn II. On the other hand, the CLOUDY calculation overestimates the observed MgI column densities by about 0.25 dex for $\log n_{\mathrm{H}}$ that concerns us. There seems to be no consistent value of $\log n_{\mathrm{H}}$. This discrepancy suggests that the input, including the background radiation field, may not fully capture the relevant physics for $\mathrm{Mg} \mathrm{I}$, as also noted by Prochaska et al. (2017). The major components, Si II, Fe II, Cr II and Ni II, vary little against $n_{\mathrm{H}}$ in so far 


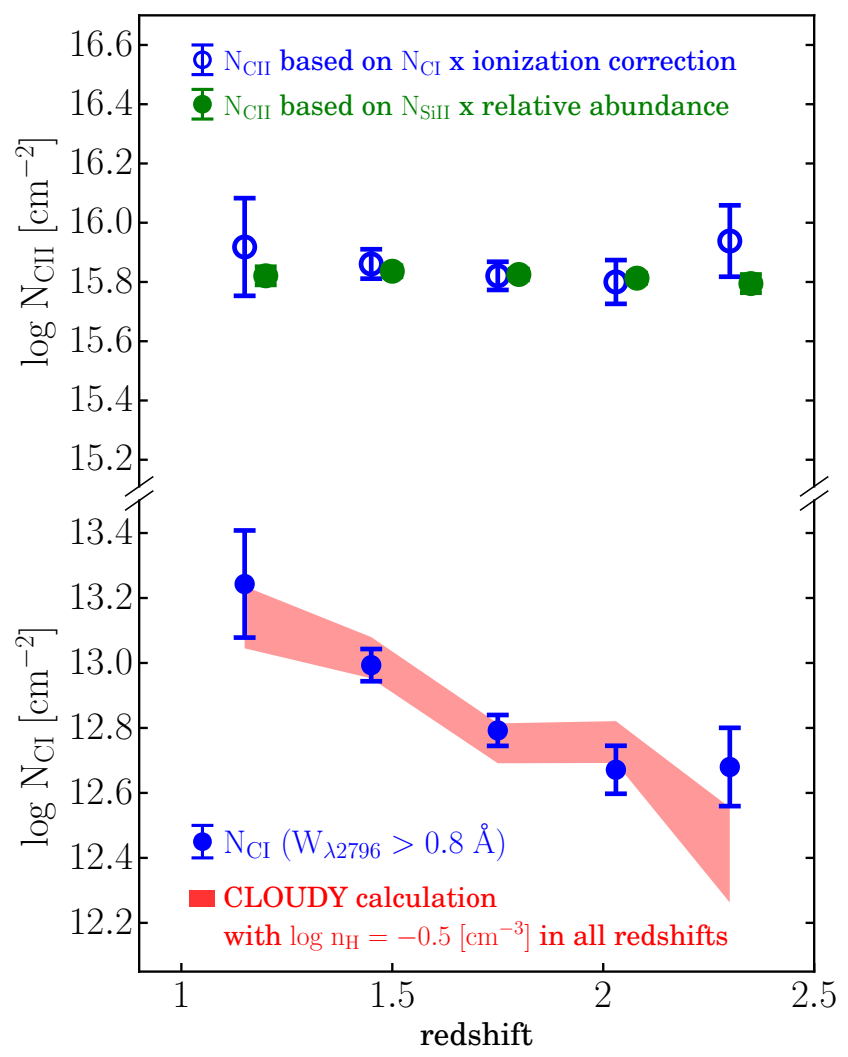

Figure 6. Redshift evolution of the column density of neutral carbon in MgII absorbers: observed (blue symbol) versus CLOUDY simulation (red shades), shown in the lower part of the figure. In the upper part CII column density calculated from CI with ionisation correction factor is shown together with CII from SiII using the Milky way halo abundance composition.

as $\log n_{\mathrm{H}}>-1$ and agree with the observed values for a wide range of $\log n_{\mathrm{H}}$ with the Milky Way halo abundance composition as input.

We estimate the heavy element abundance relative to $\mathrm{Zn}$ taking into account the ionisation correction derived from CLOUDY with $\log n_{\mathrm{H}}=-0.5$, which affects notably $\mathrm{Zn}$ estimated from ZnII: $\log \mathrm{N}_{\mathrm{Zn}} \approx \log \mathrm{N}_{\mathrm{ZnII}}+0.4$. We showed the resulting $\mathrm{Z} / \mathrm{Zn}$ in Figure 3 above. After ionisation corrections for ZnII, the depletion of the iron group elements becomes (e.g., $[\mathrm{Fe} / \mathrm{Zn}] \approx[\mathrm{FeII} / \mathrm{ZnII}]-$ $0.4) \sim 0.9$ dex, rather than 0.5 dex, and that for silicon is $\sim 0.6$ dex, rather than 0.2 dex: see grey symbols in the figure and the lower part of Table 2 .

We find from CLOUDY that $n_{\mathrm{HI}} / n_{\mathrm{H}}=0.8-0.9$ for $\log n_{\mathrm{H}}=-0.5$, hydrogen predominantly being neutral. The inferred temperature of the cloud is about $2500 \mathrm{~K}$ with about $300 \mathrm{~K}$ uncertainty . The neutral fraction stays at greater than 0.5 unless $n_{\mathrm{H}}$ becomes smaller than -1.5 , for which temperature goes up to greater than 5000 $\mathrm{K}$. These results change weakly with redshift.

The derived volume density of HI has a significant implication concerning size of the MgII cloud. It implies that cloud sizes are of the order of $r_{\text {cloud }} \sim N_{\mathrm{HI}} / n_{\mathrm{HI}} \sim$ $0.03 \mathrm{kpc}$, much smaller than the size of galaxies. This is compatible with the spatial size inferred for one specific cloud, showing Si II and CII absorption features, from a gravitationally lensed quasar (Rauch et al. 1999).

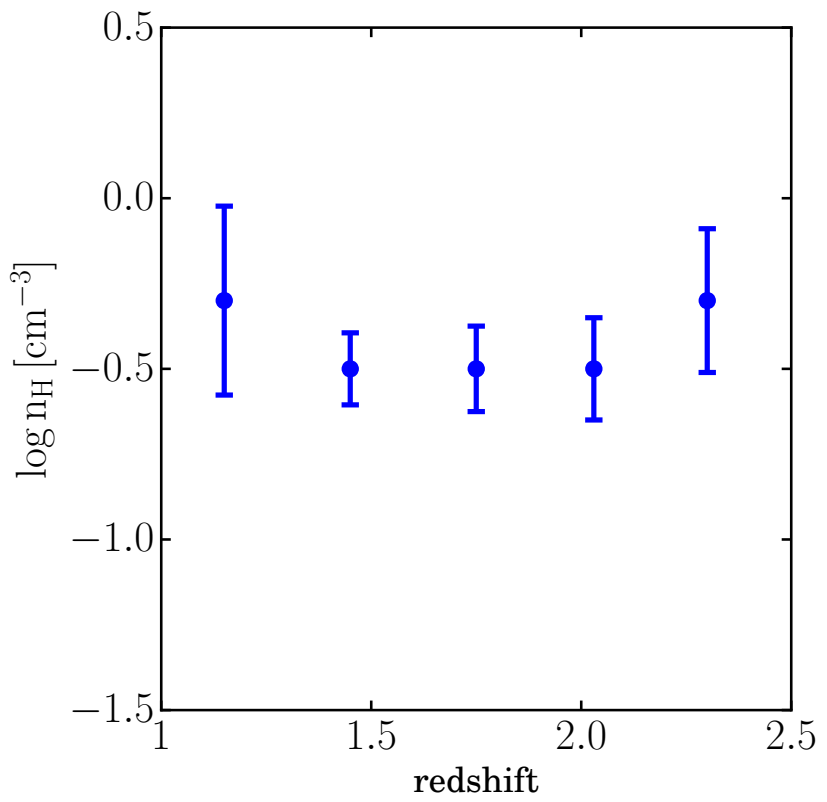

Figure 7. Optimum values of the hydrogen volume density as a function of redshift.

This is also compatible with the size of the cloud derived earlier (e.g. Rigby et al. 2002 Prochaska \& Hennawi 2009, Crighton et al. 2015). For this size we infer the typical baryonic mass of MgII clouds of the order of $M_{\text {cloud }} \sim 10^{3} \mathrm{M}_{\odot}$. Considering the fact that the covering factor of $\mathrm{Mg}$ II clouds around galaxies at redshift $\sim 0.5$ are typically 0.5 (e.g., Chen et al. 2010, Ménard \& Fukugita 2012; Nielsen et al. 2013; Lan et al. 2014), at a distance typically $20-50 \mathrm{kpc}$ from the galaxy centre. This size means that $\mathrm{Mg}$ II clouds that surround galaxies should be numerous, say, at least $10^{5}-10^{6}$. So, they are like patchy clouds or foam-like objects that surround galaxies. This is also consistent with multiple components of $\mathrm{Mg}$ II clouds in the velocity space seen in many sight lines to quasars, as observed in Churchill et al. (2003).

\subsection{Cosmic mass density of metals}

The cosmic mass density of HI in MgII clouds is estimated from

$$
\begin{array}{r}
\rho_{\mathrm{HI}}^{\mathrm{MgI}}(z)=\frac{m_{\mathrm{HI}}}{d X / d z} \int_{W_{\min }}^{\infty} d W_{\lambda 2796} \frac{d N}{d W_{\lambda 2796} d z} \times \\
N_{\mathrm{HI}}\left(W_{\lambda 2796}, z\right)
\end{array}
$$

where $d N / d W_{\lambda 2796} d z$ is the incidence rate of $\mathrm{MgII}$ absorbers (taken from Zhu \& Ménard (2013)), and $N_{\mathrm{HI}}\left(W_{\lambda 2796}, z\right)$ is the $\mathrm{Hl}$ column density we derived in equation (2): $X$ is the absorption distance. We take $W_{\min }=0.4 \AA$ as a default. We study the convergence of the integral towards the weak line limit. We find $30 \%$ decrease of $\Omega_{\mathrm{HI}}$ if $W_{\text {min }}$ is increased to $0.8 \AA$. Our extrapolation to $W_{\min }=0$ indicates the increase of the integral from the $0.4 \AA$ cutoff to be at most a few percent in $\rho_{\mathrm{HI}}$ : the integral is fairly well convergent with our default $W_{\min }$. The HI mass density obtained in equation (4) decreases towards zero redshift, as shown in Figure 


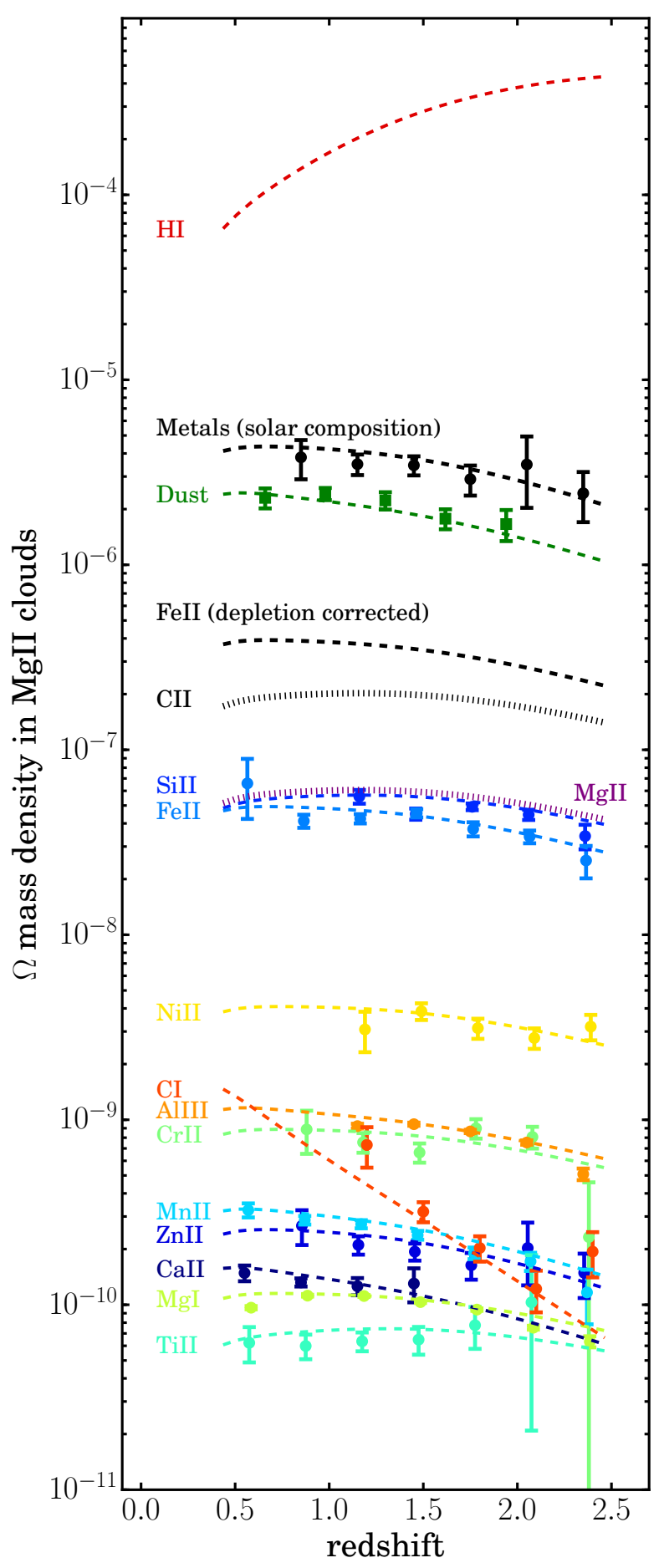

Figure 8. Cosmic mass density of heavy elements and HI borne

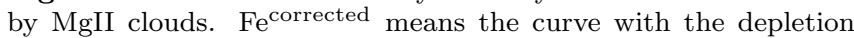
correction of 0.9 dex applied. The curve with 'metal' stands for the mass density of total heavy elements obtained from $\mathrm{Zn}$ abundance after correction for the ionisation multiplied by solar abundance of other heavy elements with respect to zinc. Other element stands for spectroscopic estimates. Dust is taken from Ménard \& Fukugita (2012). Color dashed lines show the mass densities obtained by the best-fit parameters listed in Table 1 .
8: $\Omega \simeq 4 \times 10^{-4}$ at $z=2$ decreases to $1 \times 10^{-4}$ at $z=0.5$. This estimate is consistent with Ménard \& Fukugita (2012).

We estimate the mass density of various elements in respective ionisation stages, or the density of species after the ionisation correction, by replacing HI with the relevant element and state in equation (4). In Figure 8 we show the cosmic mass density residing in the $\mathrm{MgII}$ cloud for various species, CII, MgII, SiII, FeII, NiII, CI, AlIII, CrII, MnII, ZnII, CaII, MgI and TiII. For most of the specific elements, those ionisation stages depicted in the figure stand for the predominant state in the cloud. We note that the curve for SiII is degenerated with that of MgII, including the redshift dependence. We also add a curve corrected for the depletion for Fe, total heavy element abundance (Metals) in MgII clouds taking the solar composition, and dust abundance in MgII clouds from Ménard \& Fukugita (2012).

The cosmic density of Fe from our spectroscopic analysis after the 0.9 dex depletion correction is about $\Omega_{\mathrm{Fe}}^{\mathrm{MgII}} \simeq$ $4 \times 10^{-7}$ at $z \approx 1.5$, as seen in Figure 8 . The $0.9 \mathrm{dex} \mathrm{Fe}$ depletion observed in gas phase means that about $90 \%$ of Fe $\left(\Omega_{\mathrm{Fe}}^{\text {Dust }} \sim 3.6 \times 10^{-7}\right)$ is locked in dust grains if the origin metal composition is assumed to be solar. It would be interesting to compare this amount with the estimate from dust in MgII absorbers. In Ménard \& Fukugita (2012) the amount of dust in MgII absorbers is estimated to be $\Omega_{\text {dust }}^{\mathrm{MgII}}=2.0 \times 10^{-6}(z=1.5)$ from dust reddening behind MgII absorbers using broad-band quasar photometry (green data points). With typical iron fraction $\mathrm{Fe} / \mathrm{dust} \approx 0.2^{6}$ we find $\Omega_{\mathrm{Fe}}^{\mathrm{Dust}} \approx 4 \times 10^{-7}$ in agreement with our spectroscopy-based estimate.

The increase of mass density of metals, as borne by MgII clouds is moderate towards $z=0$. We note, however, that the HI mass density is significantly decreasing towards lower redshift, by a factor of 4 from $z=2$ to $z=0.5$. This means a loss of material in Mg II clouds, say for example by their falling on galaxies or destruction for some reasons. This means that heavy elements in $\mathrm{Mg}$ II clouds are most likely lost along with HI gas. In other words, if we correct for this loss factor, the evolution of metal abundance should be traced by $\mathrm{Z} / \mathrm{H}$, rather than $\mathrm{Z}$ : the increase of the metal abundance towards lower redshift should then be significant.

The same comment also applies to the dust abundance in Ménard \& Fukugita (2012), which shows a slow evolution with redshift. The cosmic evolution of dust should be obtained by dividing their values with the mass density of HI, correcting for the gas mass loss in Mg II clouds towards low redshift.

\subsection{Evolution of the global metal abundance}

We study the metallicity evolution traced by MgII absorbers. Namely the measure of metallicity, Z/H, stands for the abundance of heavy element that would be contained in MgII clouds. When a correction is taken into account for the redshift evolution of hydrogen mass density in MgII clouds, we are led to the global abundance of metals.

The resulting $[\mathrm{Z} / \mathrm{H}]$ (denoted by blue solid circles in

6 We take dust to consist of $70 \%$ astronomical silicate and $30 \%$ graphite (e.g. Draine 2011). 


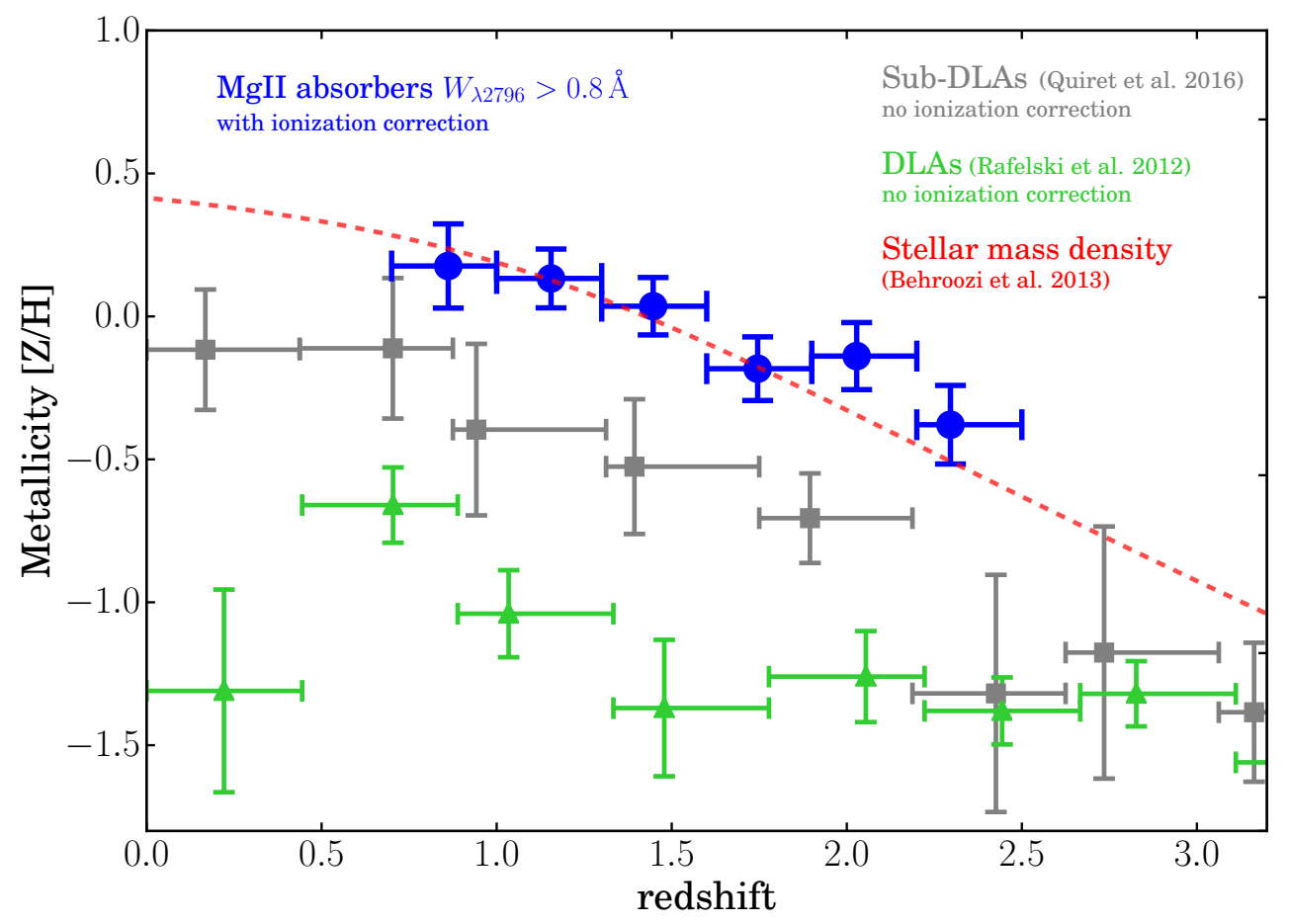

Figure 9. Metallicity $[\mathrm{Z} / \mathrm{H}]$ evolution. Metallicity of MgII clouds is shown with blue solid symbol. Metallicity of DLA is shown by green symbols (Rafelski et al. 2012). Sub-DLA (Quiret et al. 2016) is represented with grey squares. Dotted (red) curve shows the cumulative amount ot stars born drawn with an arbitrary normalisation.

Figure 9) evolves from redshift 2.5 to 0.5 , increasing by a factor of 4 . This quantity can be interpreted as ordinary metallicity $[\mathrm{Z} / \mathrm{H}]$. It reaches close to solar, in fact, approximately twice the solar metallicity at zero redshift.

In this figure we compare $[\mathrm{Z} / \mathrm{H}]$ of $\mathrm{Mg}$ II absorbers with other cool gas absorbers. The similar pattern of the evolution seen with MgII absorbers is seen in that of DLAs (green points) (Rafelski et al. 2012) and of subDLAs (grey points) Quiret et al. 2016) to note that the ionisation correction was not usually applied to the data of DLA and sub-DLA. The correction would raise those curves approximately by 0.4 dex for sub-DLA (see also Som et al. 2015), for which we expect the physical condition is similar to Mg II clouds. In our result of $\mathrm{MgII}$ the ionisation correction, most importantly that for ZnII, is included that shifted the curve upwards by about 0.4 dex.

Metallicity of $\mathrm{Mg}$ II clouds, with or without the ionisation correction, is larger than that of DLA. It is only about 0.2 dex larger than metallicity of sub-DLA, but larger by $\approx 1$ dex than that of DLA. This approximately agrees with Fukugita \& Ménard (2015), which shows that metallicity is inversely proportional to $N_{\mathrm{HI}}$ in DLA.

We show in this figure the cumulative amount of fuel used for the star formation rate in galaxies (the star formation rate is taken from Behroozi et al. (2013) integrated from a high to the relevant redshift (red dashed line) with an arbitrary normalisation. It is interesting

7 In this figure all errors are by bootstrapping. For DLA and subDLA data points we estimate the median metallicity of their samples with bootstrapping error bars of metallicity instead of the hydrogen weighted metal abundances. to observe that $[\mathrm{Z} / \mathrm{H}]$ of $\mathrm{MgII}$ clouds, i.e. what traces the total amount of metals in intergalactic media, closely traces the cumulative fuel consumed to that redshift. Metals are produced in stellar evolution: if a constant fraction of metals produced in stars is transported to intergalactic space by the galactic wind, it will contaminate circumgalactic space. Hence, it is natural to suppose that metallicity in circumgalactic space, and so in $\mathrm{Mg}$ II clouds, is proportional to cumulative star formation in galaxies (Ménard \& Fukugita 2012).

We note that the metal abundance shown in Figure 8 above means that contained in $\mathrm{Mg}$ II clouds. We must correct for the loss of gas mass in the Mg II clouds towards low redshift to obtain the global metal density evolution. Namely we must divide each component of $\Omega_{i}$ by $\Omega_{\mathrm{HI}}$.

\section{SUMMARY}

Large data bases of quasar absorption lines have enabled a spectroscopic study of the elemental abundance by stacking weak, unsaturated lines, such as Fe II, Zn II, C I, Si II, Ni II and so on, of many quasars as a function of line strengths of the Mg II lines but also of redshift.

We find that the abundance pattern of $\mathrm{Mg}$ II clouds resembles that of Galactic halo or of SMC, but it differs significantly from solar or that of Galactic interstellar matter, as has been inferred from the extinction curve (York et al. 2006, Fukugita \& Ménard 2015). We find, however, that the total abundance of heavy elements is larger than that of SMC and is close to that of Milky Way. We also confirm that iron group elements are significantly (approximately 0.9 dex) depleted in cloud spectra, as is known in interstellar gas or in DLA (van Steen- 
berg \& Shull 1988; Pettini et al. 1994). On the other hand, depletion of zinc is, if any, not significant, since the zinc abundance, or when it is multiplied with $\mathrm{Z} / \mathrm{Zn}$, is extrapolated to solar at $z=0$. Therefore, we have taken zinc as our reference for metals, in agreement with earlier reports, however after the ionisation correction for $\mathrm{Zn}$ II is taken into account. The ratios, $\mathrm{Fe} / \mathrm{Zn}, \mathrm{Si} / \mathrm{Zn}$, etc., which are consistently smaller than solar due to depletion, evolve weakly with redshift.

We find a significant evolution of metallicity $\mathrm{Z} / \mathrm{H}$ in MgII clouds from our highest $z=2.5$ to the lowest $z=0.5$, an increase by a factor of 4 . We find that this is mainly caused by a decrease of total HI column density or the HI abundance of clouds towards lower redshift. We argue that the evolution of cosmic metal abundance reflects in $\mathrm{Z} / \mathrm{H}$ with the denominator taking account of the evolution of neutral hydrogen in Mg II clouds. Figure 9 shows that the evolution of $\mathrm{Z} / \mathrm{H}$ closely traces the cumulative amount of hydrogen fuel used for star formation in galaxies: evolution of metals in $\mathrm{Mg}$ II clouds reflects star formation of galaxies.

Among the heavy elements we studied the species that shows a rapid evolution is $\mathrm{C} \mathrm{I}$, which exhibits an increase by a factor of a dex towards a low redshift $(z=0.5)$ in its column density, caused mainly by the decrease of the ionising radiation. This accounts for a rapid increase of the CI cloud incidence towards low redshifts reported in Ledoux et al. (2015).

Net HI gas bound in the MgII clouds evolves from $\Omega_{\mathrm{HI}}=4 \times 10^{-4}$ at $z=2$ to $1 \times 10^{-4}$ at $z=0.5$. We also estimate the mass density of various elements. In addition, we show that the iron abundance in dust inferred from the depletion in gas phase $\Omega \approx 4 \times 10^{-7}$ agrees with the iron abundance inferred from dust in $\mathrm{Mg}$ II clouds using reddening of quasars behind the cloud.

With the aid of the CLOUDY code we infer that the volume density of the gas is roughly $0.3 \mathrm{~cm}^{-3}$, which does not vary with redshift. This conclusion rests on the validity of CLOUDY calculations, but we are convinced with its reliability from the fact that it infallibly gives reasonable elemental abundance in so far as we have tested. In particular, the carbon abundance estimated from largely redshift-dependent CI becomes consistent with a constant after the use of the ionisation correction factor of CLOUDY, and the carbon abundance agrees with the predominant component CII, inferred from the SiII abundance. Moreover, with this density, the evolution of the CI column density as given by CLOUDY shows nearly a perfect match with that observed in our redshift range. The $n_{\mathrm{H}}$ dependence of ZnII, and Al III also match well between CLOUDY and the observation with this gas density, and we find $n_{\mathrm{H}} \approx 0.3 \mathrm{~cm}^{-3}$ is a compromise to account for ZnII and Al III.

The abundances in single ionized metal elements, such as $\mathrm{Si}$ II, Fe II, Ni II, etc, are also in a good agreement between CLOUDY and our observation with the Milky Way halo/SMC abundance pattern. These calculations also tell us that hydrogen in the MgII clouds is predominantly $(\approx 80-90 \%)$ in the HI state and the temperature is estimated to be roughly $2500 \mathrm{~K}$. Our elemental analysis overall seems to verify a validity of CLOUDY in our problem. We stress that $\mathrm{C}$ I serves as a sensitive indicator for the physical condition.

Our derived volume density of gas $0.3 \mathrm{~cm}^{-3}$ implies, together with a typical column density of the clouds $3 \times 10^{19} \mathrm{~cm}^{-2}$, that the size of clouds being $0.03 \mathrm{kpc}$, which is compatible with earlier inference (Rauch et al. 1999, Rigby et al. 2002, Prochaska \& Hennawi 2009. Crighton et al. 2015). If this is a typical size in one dimension, numerous, say $10^{6}$, clouds are needed to explain the observational indication that the covering factor of $\mathrm{Mg}$ II clouds around galaxies amounts to $50 \%$ of the sky. This means that Mg II clouds are like foam that surrounds the galaxies. This picture would explain what was found in spectroscopic observation showing multicomponents of the cloud in many line of sight (Churchill et al. 2003). Typical baryonic mass of $\mathrm{Mg}$ II clouds is of the order of $10^{3} \mathrm{M}_{\odot}$.

We thank Brice Ménard and Guangtun Zhu, who made their MgII catalogue and absorption spectra available to us in a digital form. MF thanks Hans Böhringer and Yasuo Tanaka for the hospitality at the Max-PlanckInstitut für Extraterrestrische Physik and also Eiichiro Komatsu at Max-Planck-Institut für Astrophysik, in Garching. He also wishes his thanks to Alexander von Humboldt Stiftung for the support during his stay in Garching, and Monell Foundation in Princeton at Institute for Advanced Study. He received in Tokyo a Grantin-Aid (No. 154300000110) from the Ministry of Education. Kavli IPMU is supported by World Premier International Research Center Initiative of the Ministry of Education, Japan.

\section{REFERENCES}

Asplund, M., Grevesse, N., Sauval, A. J., \& Scott, P. 2009, ARA\&A, 47, 481

Behroozi, P. S., Wechsler, R. H., \& Conroy, C. 2013, ApJ, 770, 57 Bergeron, J., \& Boissé, P. 1991, A\&A, 243, 344

Chen, H.-W., Helsby, J. E., Gauthier, J.-R., et al. 2010, ApJ, 714, 1521

Churchill, C. W., Vogt, S. S., \& Charlton, J. C. 2003, AJ, 125, 98

Crighton, N. H. M., Hennawi, J. F., Simcoe, R. A., et al. 2015, MNRAS, 446, 18

Draine, B. T. 2011, Physics of the Interstellar and Intergalactic Medium by Bruce T. Draine. Princeton University Press,

2011. ISBN: 978-0-691-12214-4,

Ferland, G. J., Porter, R. L., van Hoof, P. A. M., et al. 2013, Rev. Mexicana Astron. Astrofis., 49, 137

Fukugita, M., \& Ménard, B. 2015, ApJ, 799, 195

Haardt, F., \& Madau, P. 2001, Clusters of Galaxies and the High

Redshift Universe Observed in X-rays,

Haardt, F., \& Madau, P. 2012, ApJ, 746, 125

Lan, T.-W., Ménard, B., \& Zhu, G. 2014, ApJ, 795, 31

Ledoux, C., Noterdaeme, P., Petitjean, P., \& Srianand, R. 2015, A\&A, 580, A8

Matejek, M. S., Simcoe, R. A., Cooksey, K. L., \& Seyffert, E. N. 2013, ApJ, 764, 9

Ménard, B., Nestor, D., Turnshek, D., et al. 2008, MNRAS, 385, 1053

Ménard, B., \& Chelouche, D. 2009, MNRAS, 393, 808

Ménard, B., \& Fukugita, M. 2012, ApJ, 754, 116

Morton, D. C. 2003, ApJS, 149, 205

Nielsen, N. M., Churchill, C. W., \& Kacprzak, G. G. 2013, ApJ, 776,115

Pâris, I., Petitjean, P., Ross, N. P., et al. 2017, A\&A, 597, A79

Pettini, M., Smith, L. J., Hunstead, R. W., \& King, D. L. 1994, ApJ, 426, 79

Prochaska, J. X., \& Hennawi, J. F. 2009, ApJ, 690, 1558

Prochaska, J. X., Werk, J. K., Worseck, G., et al. 2017, ApJ, 837, 169

Quiret, S., Péroux, C., Zafar, T., et al. 2016, MNRAS, 458, 4074 

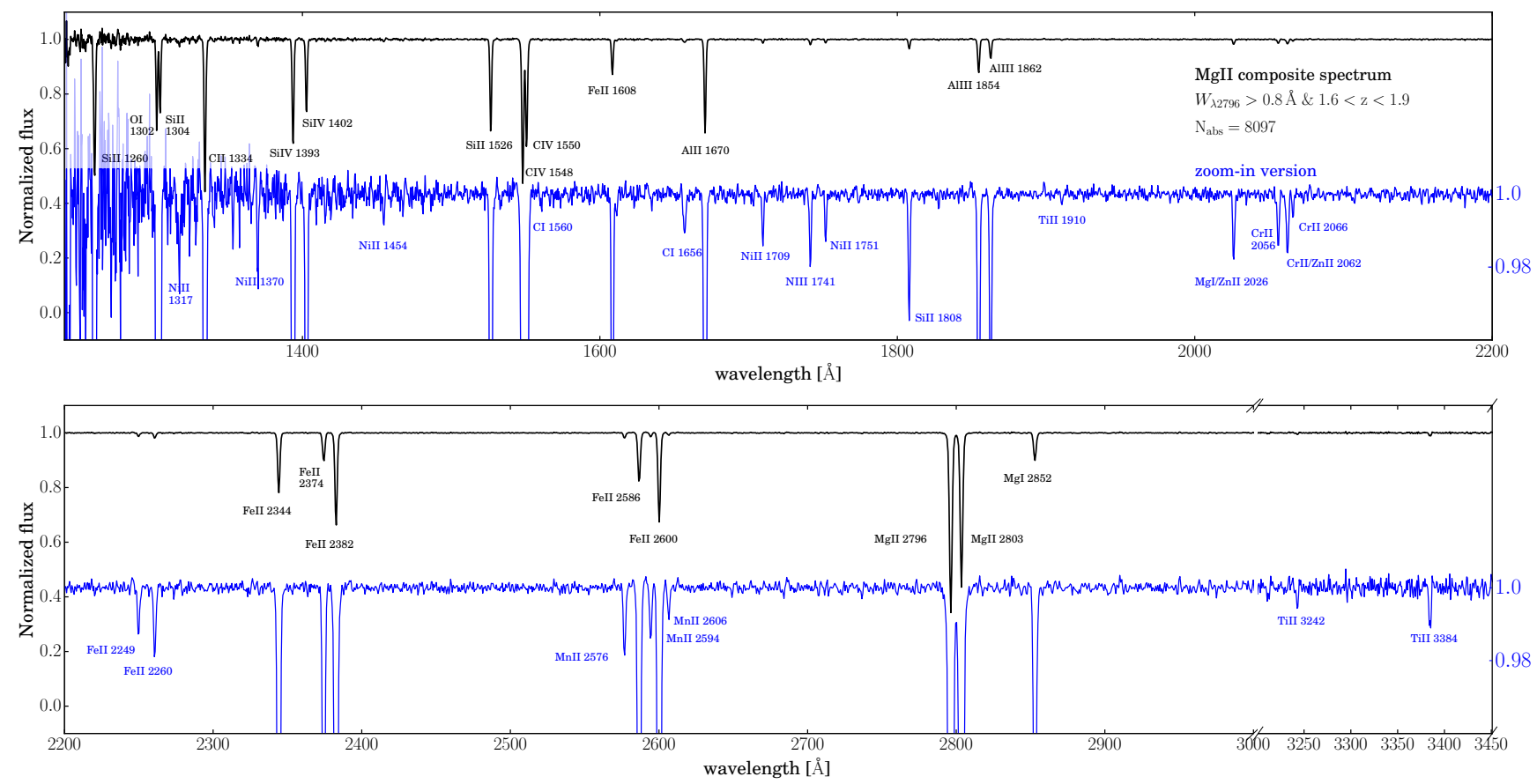

Figure 10. Example of composite spectrum. It is obtained by combining 8097 individual spectra from MgII absorbers at redshift $1.6<z<1.9$ and $W_{\lambda 2796}>0.8 \AA$. The black and blue spectra are identical and shown to emphasize absorption features in two scales. The y-axis scales for black and blue spectra are shown on the left and right, respectively.

Rafelski, M., Wolfe, A. M., Prochaska, J. X., Neeleman, M., \& Mendez, A. J. 2012, ApJ, 755, 89

Rauch, M., Sargent, W. L. W., \& Barlow, T. A. 1999, ApJ, 515, 500

Rao, S. M., Turnshek, D. A., \& Nestor, D. B. 2006, ApJ, 636, 610 Rao, S. M., Turnshek, D. A., Sardane, G. M., \& Monier, E. M. 2017, MNRAS, 471, 3428

Rigby, J. R., Charlton, J. C., \& Churchill, C. W. 2002, ApJ, 565, 743

Schneider, D. P., Richards, G. T., Hall, P. B., et al. 2010, AJ, 139,2360

Som, D., Kulkarni, V. P., Meiring, J., et al. 2015, ApJ, 806, 25

Steidel, C. C., Dickinson, M., \& Persson, S. E. 1994, ApJL, 437, L75 van Steenberg, M. E., \& Shull, J. M. 1988, ApJ, 330, 942

Welty, D. E., Lauroesch, J. T., Blades, J. C., Hobbs, L. M., \& York, D. G. 2001, ApJL, 554, L75

Wild, V., Hewett, P. C., \& Pettini, M. 2006, MNRAS, 367, 211

York, D. G., Adelman, J., Anderson, J. E., Jr., et al. 2000, AJ, 120,1579

York, D. G., Khare, P., Vanden Berk, D., et al. 2006, MNRAS, 367, 945

Zhu, G., \& Ménard, B. 2013, ApJ, 770, 130

\section{APPENDIX}

This appendix describes the details of our analysis discussed in Section 3.1. To detect and measure weak unsaturated lines, we first make median composite spectra by grouping the sample in absorber redshift and $W_{\lambda 2796}$ bins. Figure 10 shows a composite spectrum obtained by combining 8097 individual spectra from absorbers at $1.6<z<1.9$ and with $W_{\lambda 2796}>0.8 \AA$. We show two plots, black the composite spectrum and blue the same plot with an enlarged scale, with the scales shown on the left and right, respectively. We note that the signal to noise ratio of the composite spectrum is about 500-1000 and this high $\mathrm{S} / \mathrm{N}$ enables us to detect weak metal lines as labeled in the figure.

The rest equivalent widths of metal lines are measured from composite spectra with Gaussian profile fittings, as our fits shown in Figure 11. Isolated metal lines are measured with a single Gaussian profile fit, while for wavelength regions with multiple lines we adopt multiple Gaussian profiles to simultaneously fit all lines in the vicinity.

Finally, in Figure 12, we show the measured rest equivalent widths of all the metal absorption lines grouped with $W_{\lambda 2796}$ as a function of redshift. The rest equivalent widths of the metal lines increase with $W_{\lambda 2796}$ (from cyan to purple) and evolve modestly with redshift (except for CI lines). We describe these dependences with Equation 3 and show the best-fit functions with colour lines in the figure. The corresponding best-fit parameters are listed in Table 1 and shown in Figure 2 in the main text. 

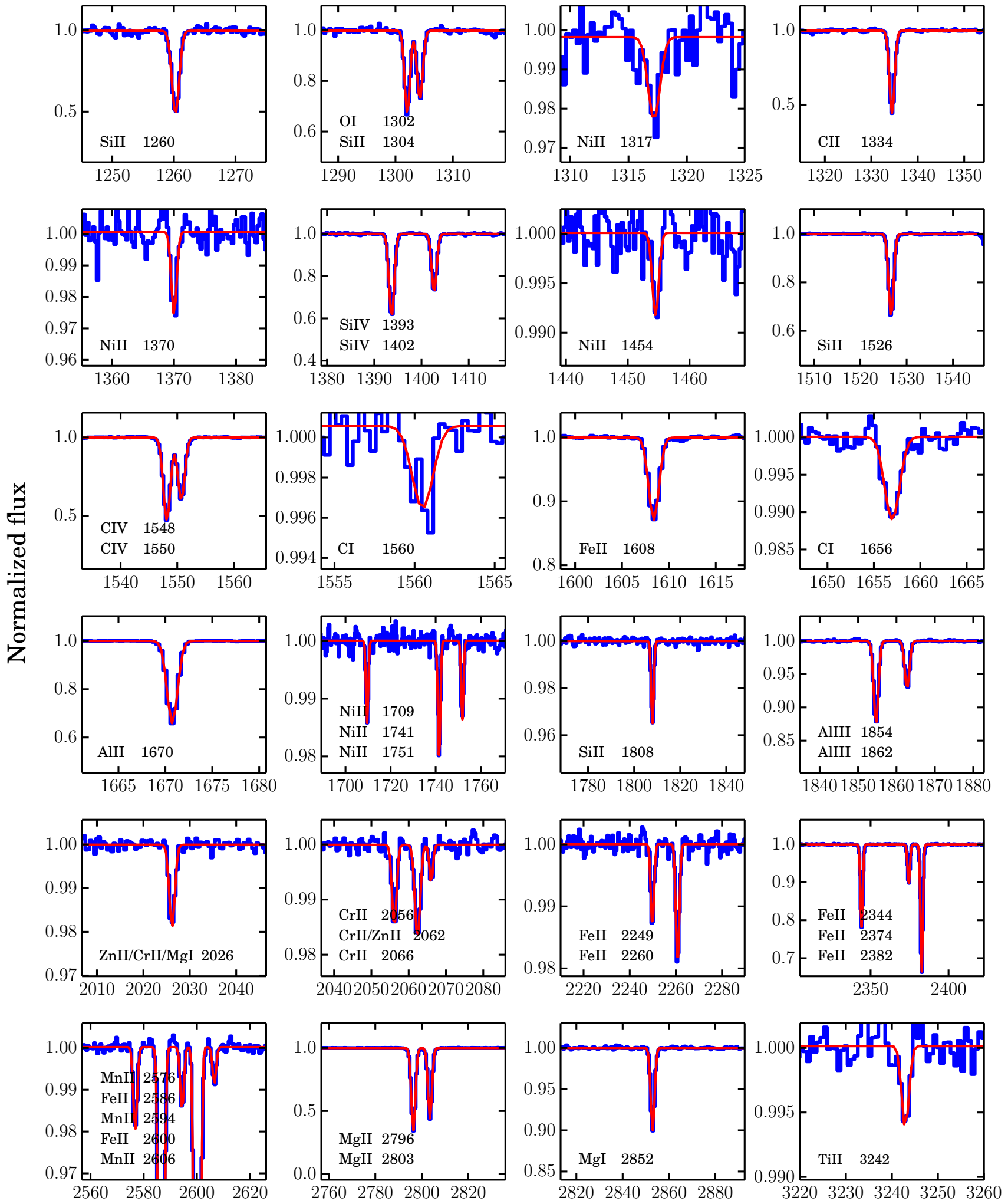

wavelength $[\AA]$

Figure 11. Example of the gaussian fittings for metal absorption lines. The observed composite spectrum is in blue and the red curves are the best-fit Gaussian profiles. 


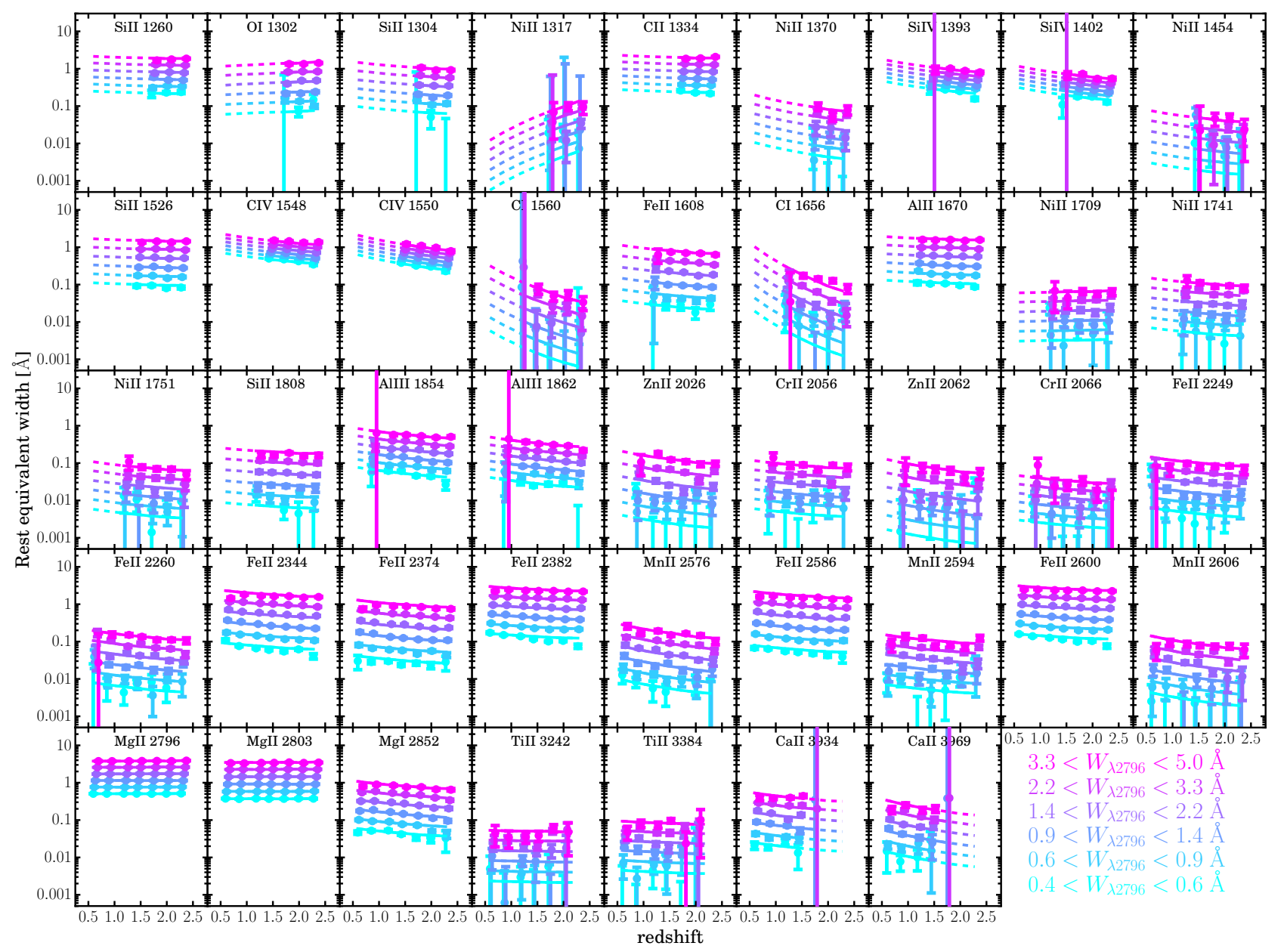

Figure 12. Rest equivalent widths of 43 metal absorption lines as a function of redshift and $W_{\lambda 2796}$. The color indicates $W_{\lambda 2796}$ from weak (cyan) to strong (purple). Best-fit functions with Equation 3 are shown with color lines and the parameter values are listed in Table 1 . 